\title{
A FIXED POINT THEOREM FOR PSEUDO-ARCS AND CERTAIN OTHER METRIC CONTINUA
}

\author{
O. H. HAMILTON
}

E. E. Moise has defined a pseudo-arc $[1]^{1}$ and has shown that it has the property of being homeomorphic to each of its nondegenerate subcontinua. Bing has shown that a pseudo-arc is homogeneous [2]. F. B. Jones, in a letter to the author, has raised the question as to whether a pseudo-arc has the fixed point property with respect to continuous transformations. It is the purpose of this note to show that a pseudo-arc is a member of a more general class of metric continua which have the fixed point property.

We shall use Moise's definition of a chain, namely: a chain $Y$ is a collection of mutually exclusive open sets (called links), $y_{1}, y_{2}, y_{3}, \cdots$, $y_{k}$, such that $y_{i}$ and $y_{j}$ have a boundary point in common if and only if $i$ and $j$ are identical or consecutive integers. If $Y$ designates a chain, $C(Y)$ will designate the closure of the set of points each of which is in a link of the chain $Y$, and $\Delta Y$ will designate the maximum diameter of a link of the chain $Y$. For convenience the closure of a link, $C\left(y_{i}\right)$, of $Y$ will be called a closed link of $Y$. A closed link $C\left(y_{i}\right)$ of $Y$ will be said to precede $C\left(y_{j}\right)$ in $Y$ if $i<j$ and to follow $C\left(y_{j}\right)$ if $i>j$.

Theorem. Let $Y_{1}, Y_{2}, Y_{3}, \cdots$ be a sequence of chains such that: (1) $C\left(Y_{1}\right)$ is a compact nonvacuous metric space, (2) $C\left(Y_{i+1}\right)$ is a subset of $C\left(Y_{i}\right)$ for each $i,(3) \operatorname{limit}_{i \rightarrow \infty} \Delta Y_{i}=0$. Let $M$ designate the continuum which is the intersection of the sets $C\left(Y_{i}\right)$. Then if $T$ is a continuous transformation of $M$ into a subset of itself, there is a point $p$ of $M$ such that $T(p)=p$.

Proof. Let $\epsilon$ be a positive real number. Let $m$ be a positive integer such that $\Delta Y_{m}$ is less than $\epsilon$. Let $A$ be the subset of $M$ consisting of all points $p$ of $M$ such that either each of the closed links of $Y_{m}$ which contain $T(p)$ follows all the closed links of $Y_{m}$ which contain $p$ or such that $T(p)$ is contained in some closed link of $Y_{m}$ which contains $p$. Let $B$ be the subset of $M$ consisting of all points $p$ of $M$ such that either each closed link of $Y_{m}$ which contains $T(p)$ precedes all the closed links of $Y_{m}$ which contain $p$ or such that $T(p)$ is contained in some closed link of $Y_{m}$ which contains $p$. The points of $M$ in the first closed link of $Y_{m}$ are in $A$ and the points of $M$ in the last

Presented to the International Congress of Mathematicians, September 6, 1950; received by the editors December 28, 1949.

${ }^{1}$ Numbers in brackets refer to the references cited at the end of the paper. 
closed link of $Y_{m}$ are in $B$, and hence these sets are nonvacuous. It is easily shown that $A$ and $B$ are closed. Then the compact continuum $M$ is the sum of the two closed sets $A$ and $B$, which therefore have a point $q$ in common. Hence $q$ and $T(q)$ lie together in some closed link $z$ of $Y_{m}$, and the distance from $q$ to $T(q)$ is less than $\epsilon$. Since $\epsilon$ is an arbitrary positive number, and since $T$ is continuous and $M$ is closed, it follows that for some point $p$ of $M, T(p)=p$.

CoRollary. $A$ pseudo-arc has the fixed point property for continuous transformations.

Proof. A pseudo-arc is a point set satisfying the definition of the set $M$ of the theorem.

It should be noted that the theorem applies to certain indecomposable continua which are not pseudo-arcs. An example is the one given by Brouwer [3] and cited by Urysohn [4].

\section{REFERENCES}

1. E. E. Moise, $A n$ indecomposable plane continuum which is homeomorphic to each of its non-degenerate subcontinua, Trans. Amer. Math. Soc. vol. 63 (1948) pp. 581594.

2. R. H. Bing, A homogeneous indecomposable plane continuum, Duke Math. J. vol. 15 (1948) pp. 729-742.

3. L. E. J. Brouwer, Zur Analysis Situs, Math. Ann. vol. 68 (1910) pp. 422-434.

4. Paul Urysohn, Memoire sur les multiplicites cantoriennes, Fund. Math. vol. 8 (1926) pp. 225-251.

Okzahoma A. and M. College 